\title{
The reciprocal relationship between physical activity and depression: Does age matter?
}

\author{
S. Wassink-Vossen ${ }^{\mathrm{a}, *}$, R.M. Collard ${ }^{\mathrm{b}}$, B.W. Penninx ${ }^{\mathrm{c}}$, S.A. Hiles ${ }^{\mathrm{d}}$, R.C. Oude Voshaar ${ }^{\mathrm{e}}$, \\ P. Naarding ${ }^{\mathrm{a}}$ \\ a Department of Old-Age Psychiatry, GGNet Apeldoorn, Zutphen, The Netherlands \\ ${ }^{\mathrm{b}}$ Department of Psychiatry, Radboud University Medical Center, Nijmegen, The Netherlands \\ ${ }^{\mathrm{c}}$ Department of Psychiatry and EMGO+ Institute for Health and Care Research, VU University Medical Center, Amsterdam, The Netherlands \\ d School of Medicine and Public Health, Faculty of Health, University of Newcastle, Callaghan, Australia \\ e University of Groningen, University Medical Center Groningen, University Center for Psychiatry, Groningen, Netherlands
}

\section{A R T I C L E I N F O}

\section{Article history:}

Received 25 September 2017

Received in revised form 21 December 2017

Accepted 30 December 2017

Available online 3 March 2018

\section{Keywords:}

Depression

Physical activity

Ageing

Mental health

Cohort study

\begin{abstract}
A B S T R A C T
Background: The level of physical activity (PA) and the prevalence of depression both change across the lifespan. We examined whether the association between PA and depression is moderated by age. As sense of mastery and functional limitations have been previously associated with low PA and depression in older adults, we also examined whether these are determinants of the differential effect of age on PA and depression.

Methods: 1079 patients with major depressive disorder (aged 18-88 years) were followed-up after twoyears; depression diagnosis and severity as well as PA were re-assessed. Linear and logistic regression analyses were used to test reciprocal prospective associations between PA and depression outcomes. In all models the interaction with age was tested.

Results: PA at baseline predicted remission of depressive disorder at follow-up (OR $=1.43$ [95\% CI: 1.07 1.93], $\mathrm{p}=.018)$. This effect was not moderated by age. PA predicted improvement of depression symptom severity in younger $(B=-2.03 ; S E=.88 ; p=.022)$, but not in older adults $(B=2.24 ; S E=1.48$; $\mathrm{p}=.128)(\mathrm{p}=.015$ for the interaction PA by age in the whole sample). The level of PA was relatively stable over time. Depression, sense of mastery and functional limitation were for all ages not associated with PA at follow-up.

Conclusions: Age did not moderate the impact of PA on depressive disorder remission. Only in younger adults, sufficient PA independently predicts improvement of depressive symptom severity after twoyear follow-up. Level of PA rarely changed over time, and none of the determinants tested predicted change in PA, independent of age.
\end{abstract}

(c) 2018 Elsevier Masson SAS. All rights reserved.

\section{Introduction}

Physical activity (PA) is an important and potentially modifiable determinant of healthy ageing with positive effects on healthrelated quality of life [1]. It is often assumed that depression increases with age, but current epidemiological studies point to a lower prevalence of depressive disorder at higher age, compared to younger age patients [2]. Population-based studies have provided strong evidence that PA decreases the risk for depressive symptoms in younger [3] as well as in older adults [4]. As the

\footnotetext{
* Corresponding author at: GGNet Department of Old-Age Psychiatry, P.O Box 2003, 7230 GC, Warnsveld, The Netherlands.

E-mail address: s.wassink@ggnet.nl (S. Wassink-Vossen).
}

majority of studies rely on (self-report) depressive symptom scales, it remains unknown whether these findings can be extrapolated to patients experiencing depressive disorder according to DSM-criteria [5,6].

To our knowledge, four longitudinal observational studies on the amount of PA in clinically depressed patients have been conducted in three different samples [7-10]. Low PA as well as less sports activity predict unfavorable outcome of depression in depressed adults over time $[7,10]$, with a reduction in the effect of low PA on depression outcome with increasing age up to 60 years [7]. In younger depressed adults, increasing levels of PA three weeks after admission was associated with decreasing levels of depressive symptoms [9]. Another sample of depressed patients aged 60 years and over, showed a lower level of PA during a depressive episode compared to their non-depressed counterparts 
[11]. In this study, no relationship between PA and the course of the depressive disorder was observed over time [8]. These findings suggest there may be a moderating effect of age on the reciprocal association between PA and the course of a depressive disorder.

PA might be useful as an (adjunct) intervention in the treatment of depressive disorder [12]. Meta-analyses on exercise interventions suggest that exercise may have a small, short term antidepressant effect in patients with depressive disorder [13]. However, it is also known that the most important preconditions for successful implementation of such interventions - participating and maintaining - are difficult to achieve, because of the nature of depressive disorder [14]. Interestingly, meta-regression showed that studies restricted to adults over 60 years of age showed a higher efficacy than those with subjects below 60 years [15]. This contrasts with above described results of observational studies in which the association between PA and depression decreases with age $[7,8]$. This discrepancy might be explained by selection bias. Patients participating in intervention studies may have less functional limitations and a higher sense of mastery compared to patients participating in observational studies. Functional limitations increase [16] and sense of mastery decreases with age [17]. Previous research in the same cohort used in the current study demonstrated that limitations in daily functioning in different life domains and a lower sense of mastery - the understanding that individuals hold about their ability to control the circumstances of their lives- were the only two significant explanatory factors for the difference in PA between depressed and non-depressed older adults among multiple factors [11]. In light of the discrepancy in findings between observational and interventional studies and the less favorable course of depression with increasing age $[18,19]$, it is important to know more about the moderating role of age in the relationship between PA and course of depression. To our knowledge, this has never been studied before across the entire adult lifespan within one study. This knowledge will contribute to determining whether age-specific elements are needed in the development of interventions aimed at increasing PA in patients experiencing depressive disorders.

This study has been designed to examine whether age moderates the reciprocal relationship between PA and the course of depressive disorder over two years in people with depressive disorders at baseline. We expect that the effect of PA on depression outcome after two years and the effect of depression on change in PA diminishes with increasing age. In addition, we will explore whether sense of mastery and functional limitations are potential determinants of an increase in PA at follow-up for all ages.

\section{Methods}

\subsection{Study sample}

Data were obtained from the baseline and two-year assessment of the Netherlands Study of Depression and Anxiety (NESDA; participants aged 18-65 years) and the Netherlands Study of Depression in Older Persons (NESDO; participants aged 60-93 years); two ongoing, multi-centre cohort studies with similar methods and infrastructures that examine the natural course, determinants, and consequences of depression [20,21]. Assessments included written questionnaires, interviews, a medical examination, cognitive tests and collection of blood and saliva samples. From 2004 until 2007, the initial NESDA sample was recruited and consisted of 2981 persons with a current depressive and/or anxiety disorder $(n=1701)$, a remitted depressive and/or anxiety disorder $(n=628)$, or no lifetime depressive and anxiety disorder $(n=652)$. Participants were recruited from the general population, primary healthcare, and outpatient mental health care facilities. From 2007 until 2010 the initial NESDO sample was recruited, and this sample consisted of 510 older adults (60 years and older) with either a current depressive disorder $(n=378)$ or no lifetime depressive disorder $(n=132)$, recruited through primary healthcare and out- and inpatient mental healthcare facilities. In both studies, a current psychiatric diagnosis was defined by a recency of 6 months. Exclusion criteria for both cohorts were: insufficient command of the Dutch language or insufficient capability to participate, a primary clinical diagnosis of a psychiatric disorder other than depressive and anxiety disorders, and for NESDO additionally: (clinician-suspected) dementia or a Mini- Mental State Examination (MMSE) score $<18$ (out of 30) [22]. For the current study, baseline and two-year follow-up data were used and participants were included when the following criteria were met: a current major depressive disorder diagnosis at baseline; a valid scale score for the short form self-report International Physical Activity Questionnaire (IPAQ) and participation at two-year follow-up. This selection resulted in 1079 participants being included in this study (see Fig. 1). Baseline faceto-face assessments were completed at participating centers between 2004 and 2007 for NESDA, and between 2007 and 2010 for NESDO. Ethical approval was obtained by both NESDA and NESDO from all ethical review boards of the participating centers, and all participants provided informed consent.

\subsection{Measures}

\subsubsection{Depression}

The data on major depressive disorder diagnosis were obtained from CIDI version 2.1, developed by the World Health Organization (WHO) [23]. The CIDI is a fully structured interview that diagnoses psychiatric disorders in adults according to the criteria of DSM-IV. The WHO field trials found high inter-rater reliability (kappa: 0.97), high test-retest reliability (kappa: 0.66) and high validity for depressive and anxiety disorders [24,25].

The severity of depression was assessed by the 30-item selfrating Inventory of Depressive Symptomatology (IDS) [26]. Each item consists of a series of four statements about the symptom. The

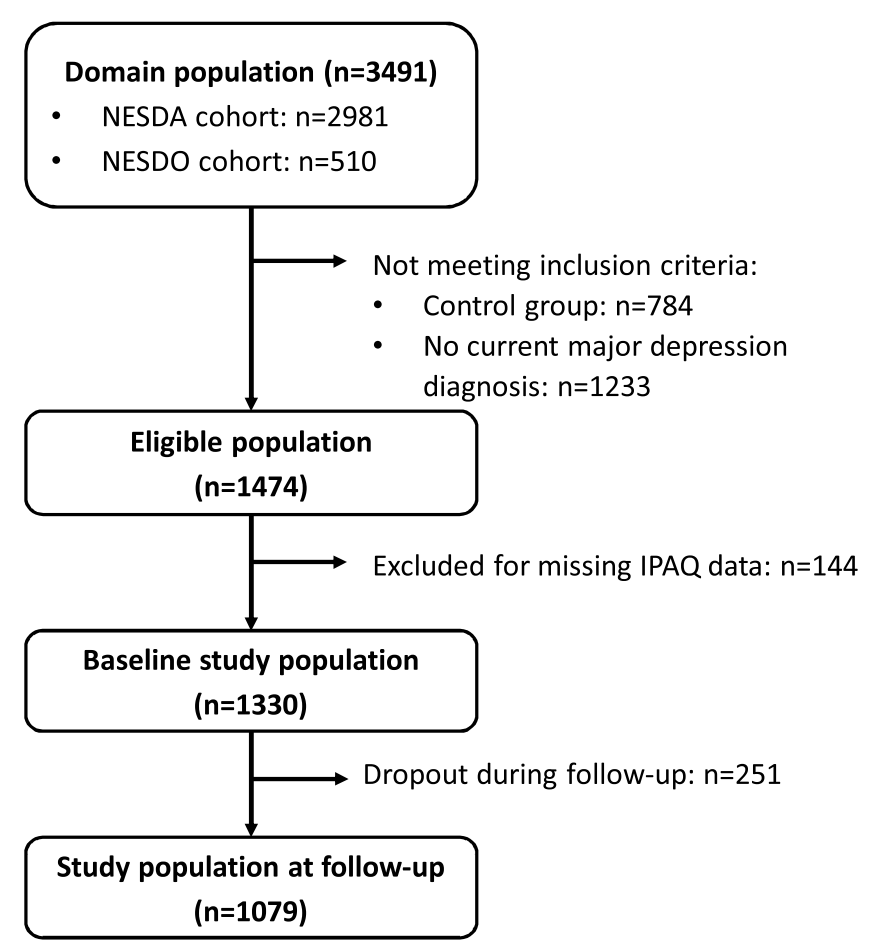

Fig. 1. Flowchart sample population. 
psychometric properties of the IDS are acceptable, with Cronbach's alpha ranging from 0.76 to 0.82 for adults with current depression [27]. Total scores were used as outcome measure. IDS change score was calculated as IDS score at two year follow up minus baseline score.

\subsubsection{Physical activity}

PA was measured with the Dutch short version International Physical Activity Questionnaire (IPAQ). This self-report questionnaire, consisting of eight items, was developed as a tool for crossnational monitoring of PA in adults based on sports and daily activities in the past week [28]. The psychometric properties of the IPAQ are acceptable for adults [28]. For older persons the criterion validity is adequate in the general population [29]. According to the official IPAQ guidelines [30], data are summed within each activity item (i.e., vigorous intensity, moderate intensity, walking) to estimate the level (low, moderate or high) of PA per week.

PA was operationalized in two ways: "sufficient" PA (yes/no) according to the WHO (primary determinant) and total amount of PA per week (for a sensitivity analysis). Patterson [30] proposed that the 'moderate' category on the IPAQ is equivalent to "half an hour of at least moderate-intensity PA on most days", the former leisure time-based PA population health recommendation by the WHO. Because of this clinically relevant classification, the three categories scored from the IPAQ were dichotomized into 'insufficient physical activity' (IPAQ category 'low') and 'sufficient physical activity' (IPAQ categories 'moderate' and 'high'). The outcome variable 'change of physical activity level' in this study applies to the participants that switch between the categories insufficient and sufficient PA in two years (i.e., 'increased' or 'decreased', with 'no change' as reference).

For the sensitivity analyses, total weekly physical activity was estimated by converting the activity items into multiples of the basal resting energy expenditure (Metabolic Equivalent of Task[MET]) [31]. The variable total MET minutes per week was initially not normally distributed and was normalized by log transformation.

\subsubsection{Covariates}

2.2.3.1. Sociodemographics. Gender (male/female), educational level in years (range 5-18 years) and partner status (respondent is married or has partner: yes/no) were obtained from general questions from the baseline examination.

2.2.3.2. Physical function. Body Mass Index (BMI) was calculated as weight in kilograms divided by the square of height. Number of chronic diseases was assessed by self-report questions about the presence of somatic diseases (cardiac diseases, cerebrovascular accident, hypertension, peripheral atherosclerosis, diabetes mellitus, chronic non-specific lung disease, liver diseases, thyroid diseases, epilepsy, intestinal diseases, arthritis/arthrosis, and cancer) [32]. Antidepressant drug treatment was based on the WHO Anatomical Therapeutic Chemical (ATC) classification system codes, three types of antidepressant were distinguished: tricyclic antidepressants (TCAs or non-selective monoamine reuptake inhibitors), selective serotonin reuptake inhibitors (SSRIs), and 'other antidepressants'. 'No use or infrequent use' (only when necessary or less than $50 \%$ of the days in a month) and 'frequent use' (more than $50 \%$ of the days in a month or daily) were distinguished.

2.2.3.3. Other lifestyle characteristics. Smoking was dichotomized into current smoker and non-smoker/former smoker. Alcohol use was scored and classified with the Alcohol Use Disorders Identification Test (AUDIT), whereby the cut-off score of $\geq 8$ points indicates hazardous and harmful alcohol use [33].

\subsubsection{Predictors of change in physical activity}

Besides depression outcome and age, sense of mastery and functional limitations were tested as predictors of change in PA. Functional limitations were measured with the WHO Disability Assessment Schedule [34]. This self-report questionnaire captures the level of functioning. In this study, total score (range 0-100) was used. Higher scores indicate more functional limitations. The WHODAS 2.0 was found to have high internal consistency with a Cronbach's alpha of 0.86 and a high test-retest reliability (intraclass correlation coefficient: 0.98) [35].

For sense of mastery, the Short Mastery Scale was used to determine the sense of mastery over one's life. Lower scores (within the range of 5-25) indicate lower sense of mastery [36,37]. The scale has shown good construct validity [38].

\subsection{Analysis}

Firstly, demographic and clinical characteristics of the study sample and participants lost to follow up were compared using independent samples $t$-tests for normally distributed, continuous variables and $\chi^{2}$ tests for categorical variables.

Then, demographic and clinical characteristics of the sample were calculated, comparing depression and PA measures at baseline and follow-up by paired-samples $t$-tests for normally distributed continuous variables, nonparametric Wilcoxon signed rank test for skewed continuous variables and McNemar's tests for categorical variables.

Next, we examined cross-sectionally whether the interaction between age and PA (sufficient PA [no/yes] and sensitivity analyses with total METmin/week) at baseline (independent variables) was associated with depressive symptom severity (dependent variable) with linear regression, adjusted for potential confounders: demographic characteristics, physical function and lifestyle characteristics.

Subsequently, to examine the association between PA (sufficient PA [no/yes] and total METmin/week) at baseline (independent variables) and depression outcome at two-year follow-up (dependent variable), fully adjusted regression models were built. Logistic regression was used for remission (no/yes) at two-year follow-up, and linear regression was used for depressive symptom severity (total score IDS at two years follow up, corrected for baseline IDS score) at two-year follow up. In all models the interaction between age (continuous variable) and PA (sufficient yes/no dichotomous and total METmin/week continuous) was tested and when $\mathrm{p}<.1$, stratified analyses by age group ( $<60$ years and 60 years and older) were presented.

Finally, multinomial logistic regression analyses were carried out to examine whether depression outcome (i.e., diagnosis at two-year no/yes or change in IDS score after two years), or determinants measured at baseline (age, functional limitation, sense of mastery) predicted change in PA outcome at two years in the following categories: increase from insufficient to sufficient PA; decrease from sufficient to insufficient PA; and no change (reference). Linear regression for sensitivity analyses used total amount of PA (total METmin/week) at two years as the dependent variable, corrected for baseline total METmin/week.

The interactions between age and depression outcome, functional limitations or mastery were tested. All models were adjusted for potential confounders: demographic variables, physical function and lifestyle characteristics.

\section{Results}

Patients lost to follow up at 2 years $(n=251)$ were less physically active, more severely depressed and had more functional limitations at baseline compared to the study sample 
of 1079 patients that participated in the two-year follow-up (all $\mathrm{p}<.05)$.

Table 1 presents the baseline characteristics and two-year depression and PA outcomes of the sample. Of the 1079 participants at two-year follow-up, 635 (58.9\%) achieved remission of the depressive disorder. Depression severity also decreased in two years with a mean difference of 9 points in IDS score $(\mathrm{p}<.001)$. After two years, 688 (73.9\%) participants did not change their level of PA, 114 (12.2\%) increased their level and 129 (13.9\%) participants decreased their level of PA. There was also an increase in total METmin/week in two years (Wilcoxon signed-rank test, $\mathrm{p}=.009$ ).

\subsection{Cross-sectional associations}

Cross-sectional linear regression models showed that sufficient PA at baseline was associated with lower scores on the IDS (fully adjusted model: $\mathrm{B}$ (unstandardized coefficient) $=-2.0$; SE (standard error) $=0.84 ; \mathrm{p}=.017$ ). The association between sufficient PA and severity of depression was not moderated by age ( $\mathrm{p}$ for interaction $=.434$ ). Similarly, the sensitivity analyses showed that a higher number of METmin/week was associated with lower scores on the IDS (fully adjusted model: $\mathrm{B}=-0.667 ; \mathrm{SE}=0.57 ; \mathrm{p}=<.001$ ). This association was not moderated by age $(\mathrm{p}=.982)$.

\subsection{Prediction of depression outcome}

\subsubsection{Remission}

Logistic regression analyses showed that sufficient PA at baseline increased the odds of remission at follow-up with an unadjusted odds ratio (OR) of 1.60 [95\% CI: 1.21-2.11] $(\mathrm{p}=.001)$ and a fully adjusted OR of 1.43 [95\% CI: 1.07-1.93] ( $\mathrm{p}=.018)$. This effect was not moderated by age $(p=.570)$. The sensitivity analyses with total amount of PA showed that a higher total
METmin/week at baseline increased the odds of remission at follow up, with an unadjusted OR of 1.07 [95\% CI: 1.01-1.14] $(\mathrm{p}=.031)$, but this effect was no longer significant in the fully adjusted model ( $\mathrm{OR}=1.02$ [95 CI: 0.95-1.08], $\mathrm{p}=.642)$ and was not moderated by age $(\mathrm{p}=.151)$.

\subsubsection{Improvement of depressive symptoms}

Linear regression models showed that reporting sufficient PA at baseline predicted lower depressive symptom severity after twoyear follow-up compared to insufficient $\mathrm{PA}(\mathrm{B}=-5.97$; $\mathrm{SE}=2.35$; $\mathrm{p}=.011$ ). As this effect was moderated by age (interaction term age by physical activity: $p=.015$ ), results were stratified by age ( $<60$ years and $\geq 60$ years). Table 2 shows that sufficient PA predicted a significant decrease of depression severity in younger adults $(\mathrm{B}=-2.03 ; \mathrm{SE}=0.88 ; \mathrm{p}=.022)$, but not in older adults ( $\mathrm{B}=2.24 ; \mathrm{SE}=1.48 ; \mathrm{p}=.128$ ).

The sensitivity analyses with total amount of PA showed that total METmin/week at baseline did not predict a change in depressive symptom severity over the two-year follow-up, neither in the unadjusted $(B=-0.050 ; S E=0.17 ; p=.766)$ nor in the fully adjusted model $(B=0.113 ; \mathrm{SE}=0.18 ; \mathrm{p}=.499)$. The relationship was not moderated by age $(\mathrm{p}=.414)$.

\subsection{Prediction of physical activity outcome}

\subsubsection{Change in (in)sufficient PA}

Separate multinomial logistic regression analyses showed that none of the potential determinants accounted for change in PA after two years. Remitted depression, change in depressive symptom severity at two years, age, mastery, and functional limitations were all not associated with a change of PA at two years. Moreover, none of these characteristics interacted with age on the prediction of change in PA at two-year follow-up (data not shown).

Table 1

Characteristics of the study population.

\begin{tabular}{|c|c|c|c|c|}
\hline \multirow[t]{2}{*}{ Characteristics } & & \multicolumn{3}{|c|}{ Population $(\mathrm{n}=1079)$} \\
\hline & & Baseline & Two year outcome & $p$ \\
\hline $\begin{array}{l}\text { Demographics: } \\
\text { - Age (years) } \\
\text { - Female sex } \\
\text { - Years of education } \\
\text { - Living status with partner (yes) }\end{array}$ & $\begin{array}{l}\text { mean }(\mathrm{SD}) \\
\mathrm{n}(\%) \\
\text { mean }(\mathrm{SD}) \\
\mathrm{n}(\%)\end{array}$ & $\begin{array}{l}47.1(16.3) \\
703(65.2) \\
11.6(3.3) \\
670(62.1)\end{array}$ & & \\
\hline $\begin{array}{l}\text { Psychopathology: } \\
\text { - Depression diagnosis (CIDI) } \\
\text { - Depression severity (IDS-score) } \\
\text { - Sense of mastery (Pearlin) }\end{array}$ & $\begin{array}{l}\mathrm{n}(\%) \\
\text { mean }(\mathrm{SD}) \\
\text { mean }(\mathrm{SD})\end{array}$ & $\begin{array}{l}1079(100) \\
31.5(12.4) \\
14.7(4.0)\end{array}$ & $\begin{array}{l}444(41.1) \\
22.6(13.0)\end{array}$ & $\begin{array}{l}<.001 \\
<.001\end{array}$ \\
\hline $\begin{array}{l}\text { Lifestyle characteristics: } \\
\text { - Sufficient physical activity (yes) } \\
\text { - Follow up change in physical activity: } \\
\text { - no change } \\
\text { - increased PA } \\
\text { - decreased PA } \\
\text { - Total amount of PA (Total METmin/week) } \\
\text { - Current smoker (yes) } \\
\text { - Harmful alcohol use (yes) } \\
\text { - Body Mass Index }\end{array}$ & $\begin{array}{l}\mathrm{n}(\%) \\
\mathrm{n}(\%) \\
\mathrm{n}(\%) \\
\mathrm{n}(\%) \\
\text { median (range) } \\
\mathrm{n}(\%) \\
\mathrm{n}(\%) \\
\text { mean (SD) }\end{array}$ & $\begin{array}{l}2457(0-19278) \\
420(38.9) \\
208(19.3) \\
25.9(5.2)\end{array}$ & $\begin{array}{l}687(73.5) \\
688(73.9) \\
114(12.2) \\
129(13.9) \\
2712(0-17892)\end{array}$ & .337 \\
\hline $\begin{array}{l}\text { Physical functioning: } \\
\text { - Number of chronic diseases } \\
\text { - Functional limitations (WHODAS) } \\
\text { - Antidepressant drug treatment: } \\
\text { - TCA } \\
\text { - SSRI } \\
\text { - Other AD }\end{array}$ & $\begin{array}{l}\text { median (range) } \\
\text { mean (SD) } \\
\mathrm{n}(\%) \\
\mathrm{n}(\%) \\
\mathrm{n}(\%)\end{array}$ & $\begin{array}{l}1.0(0-8) \\
26.6(12.5) \\
82(7.6) \\
302(28.0) \\
157(14.6)\end{array}$ & & \\
\hline
\end{tabular}

Bold value in tables means $\mathrm{P}=<.05$.

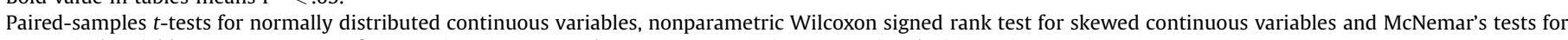
categorical variables. IDS: Inventory of Depressive Symptomatology; CIDI: Composite International Diagnostic Interview. 
Table 2

The interaction of age in the longitudinal association between physical activity and depressive symptoms (IDS) after two years.

\begin{tabular}{|c|c|c|c|c|c|c|}
\hline & \multicolumn{3}{|c|}{ Unadjusted model } & \multicolumn{3}{|c|}{ Fully adjusted model $^{\mathrm{a}}$} \\
\hline & B & SE & $p$ & B & SE & $p$ \\
\hline \multicolumn{7}{|l|}{ Main analysis (whole sample): } \\
\hline - Sufficient physical activity & -5.34 & 2.35 & .023 & -5.97 & 2.35 & .011 \\
\hline - Age & 0.04 & 0.03 & .293 & 0.013 & 0.018 & .744 \\
\hline - Age by physical activity & 0.100 & 0.046 & .028 & 0.110 & 0.045 & .015 \\
\hline \multicolumn{7}{|l|}{$\begin{array}{l}\text { Stratified analyses: } \\
\text { Impact of sufficient PA (stratified by age) }\end{array}$} \\
\hline - Sufficient PA (in patients aged $<60$ years) & -3.62 & 1.07 & .001 & -2.03 & 0.88 & .022 \\
\hline - Sufficient PA (in patients aged $>60$ years) & -0.60 & 1.75 & .733 & 2.24 & 1.48 & .128 \\
\hline
\end{tabular}

Bold value in tables means $\mathrm{P}=<.05$.

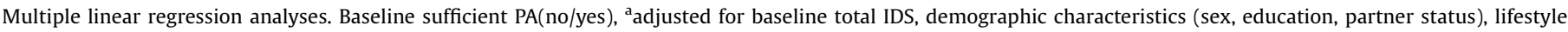

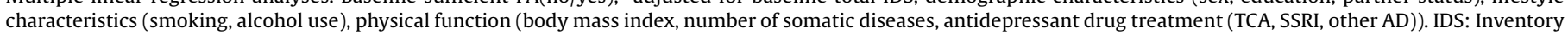
of Depressive Symptomatology.

\subsubsection{Total METmin/week}

Remission of depression, functional limitations and sense of mastery also did not predict an increase in MET-minutes over two years in the sensitivity analyses using linear regression analyses with total number of MET minutes at two year follow-up. Nonetheless, a less favorable change in depressive symptom severity over the two-year follow-up predicted a lower number of METmin/week at two years follow up, corrected for baseline total METmin/week only $(\mathrm{B}=-0.016 ; \mathrm{SE}=0.01 ; \mathrm{p}=.001)$ as well as in the fully adjusted model $(B=-0.013 ; S E=0.01 p=.007)$. In the sensitivity analyses, none of the characteristics interacted with age on the METmin/week at two year follow-up.

\section{Discussion}

Sufficient PA is - irrespective of age - an independent predictor of remission in clinically depressed patients. Age moderates the impact of PA on the improvement of depressive symptoms over time: Only in younger adults, sufficient PA independently predicts improvement of depressive symptom severity over a two-year follow-up. Another interesting finding of this study is that level of PA rarely changed over time, and none of the determinants tested predicted change in PA, independent of age. Decreased PA found in our depressed participants of all ages may therefore reflect a trait instead of a state characteristic, which will be discussed in more depth below.

The finding that sufficient PA predicts remission of depressive disorder is in line with studies on depressive symptoms in the general population [3]. Sufficient PA can thus not only be seen as a protective factor for not developing depressive disorder, but also as a predictive factor for remission of depression in clinically depressed patients of all ages. We contributed to the growing body of knowledge about the importance of sufficient PA for people at risk for or experiencing depressive disorder [13]. In younger adults, we furthermore found that sufficient PA led to a decrease of depressive symptoms after two years. In older adults, the relationship seems more complex: sufficient activity at baseline did not lead to higher reduction in depressive symptoms after two years compared to their low active counterparts. The most likely explanation is that scales for self-report of depressive symptoms in older adults measure more than just depression severity. It could, for example, also reflect the severity of comorbid somatic illnesses. Although depression showed remission in the older participants with sufficient PA, the decrease of total IDS score is small. It is known that in case of low depression severity, individual (self-report) depressive symptom items more often reflect underlying physical illness instead of reflecting psychopathology [39].
The second finding of this study is that the level of PA, whether sufficient or insufficient, rarely changed in this initially depressed group. Although longitudinal studies in the general population under 60 are scarce, the same trend is shown [40]. Studies in persons 60 years and older have mixed results. They can show stability in PA over time [41], as well as a decrease in PA over time [40]. Our results suggest that lower PA in people with depression of all ages might reflect a trait instead of a state characteristic, i.e. low PA is independent of depression state and could be a feature of one's personality. In contrast to our expectations, the stability of PA was independent of depression outcome. Neither remission of the depressive disorder, nor a decline in depressive symptoms over time predicted a change in PA at follow-up. This may not explain the small effect of PA interventions on depressive symptoms, but it may explain why these effects diminish over time [13]. Interventions for improving PA should therefore be focused on a sustained change in activities and should be incorporated in a broader multidisciplinary plan on improving health-related behavior and self-management skills [42].

This study has several strengths. First, due to the substantial sample size and the broad age range, we had sufficient power to test the interaction effects of age in models fully adjusted for possible confounders of the association between PA and depression, and vice versa. Second, our results can be generalized to a population of clinical depressed adults, as our sample reflects all different stages of depression in different healthcare settings. Finally, we not only included DSM-IV diagnosis of depression in our measures, but also a well-validated measure of symptom severity (IDS).

Nevertheless, this study has some limitations. First, although the IPAQ is a valid and widely used self-report questionnaire on PA, the validity of this scale in depressed (older) persons is unclear. People with depression tend to be negatively biased about their own performance [43]. On the other hand, self-reported physical activity is over-reported with the IPAQ [44] Validating this selfreport measures of PA in depressed subjects is warranted, but not available yet [45]. Therefore, use of accelerometers to measure PA would have been more impropriate [46].

Second, the lack of an interval assessment on PA between the two years is a limitation. Therefore, we have no information on the changes in PA over this time period.

Third, only the number of chronic health conditions was available to measure the effect of serious chronic health burden on both physical activity and depression. The use of a validated index (e.g., the Charlson Comorbidity Index) would have been more accurate and could have given specific information on the effect of individual somatic diseases on PA, depression and their interrelationship. Finally, because of the observational design of this 
study, and the lack of details on treatment modalities, we cannot cover the effects of other treatment on PA.

\section{Conclusion}

This study reveals that, although age does not moderate the impact of PA on remission of the depressive disorder, improvement of depressive symptoms was significantly lower among older compared to younger persons with sufficient PA. This might be explained by underlying somatic conditions affecting depressive symptom scores in old age. Given that PA only marginally increases over time in all age groups, the increase does not result in a significantly high proportions of patients converting from insufficient to sufficient PA. Moreover, depressive symptoms, sense of mastery and functional limitations were not determinants of increased PA. Collectively, our findings suggest that PA of depressed patients in all ages might reflect a trait instead of a state characteristic. This should be taken into account in further development of lifestyle interventions.

\section{Acknowledgements}

The infrastructure for the NESDA study (www.nesda.nl) has been funded through the Geestkracht program of the Netherlands Organisation for Health Research and Development (Zon-Mw, grant number 10-000-1002) and financial contributions by participating universities and mental health care organizations (VU University Medical Center, GGZ inGeest, Leiden University Medical Center, Leiden University, GGZ Rivierduinen, University Medical Center Groningen, University of Groningen, Lentis, GGZ Friesland, GGZ Drenthe, Rob Giel Onderzoekscentrum). The infrastructure for NESDO is funded through the Fonds NutsOhra, Stichting tot Steun VCVGZ,NARSAD The Brain and Behaviour Research Fund, and the participating universities and mental health care organizations (VU University Medical Center, Leiden University Medical Center, University Medical Center Groningen, Radboud University Nijmegen Medical Center, GGZ inGeest, GGNet, GGZ Nijmegen, GGZ Rivierduinen, Lentis, and Parnassia). All authors state that there are no commercial or financial involvements that might present a conflict of interest with the here presented article.

\section{References}

[1] Bize R, Johnson JA, Plotnikoff RC. Physical activity level and health-related quality of life in the general adult population: a systematic review. Prev Med 2007;45(December (6)):401-15.

[2] Beekman AT, Copeland JR, Prince MJ. Review of community prevalence of depression in later life. Br J Psychiatry 1999;174(April):307-11.

[3] Mammen G, Faulkner G. Physical activity and the prevention of depression: a systematic review of prospective studies. Am J Prev Med 2013;45(November (5)):649-57.

[4] Win S, Parakh K, Eze-Nliam CM, Gottdiener JS, Kop WJ, Ziegelstein RC. Depressive symptoms, physical inactivity and risk of cardiovascular mortality in older adults: the Cardiovascular Health Study. Heart 2011;97(March (6)):500-5.

[5] Blake H, Mo P, Malik S, Thomas S. How effective are physical activity interventions for alleviating depressive symptoms in older people? A systematic review. Clin Rehabil 2009;23(October (10)):873-87.

[6] Sjosten N, Kivela SL. The effects of physical exercise on depressive symptoms among the aged: a systematic review. Int J Geriatr Psychiatry 2006;21(May (5)):410-8.

[7] Boschloo L, Reeuwijk KG, Schoevers RA, Penninx B WJH. The impact of lifestyle factors on the 2-year course of depressive and/or anxiety disorders. J Affect Disord 2014:159(April):73-9.

[8] Wassink-Vossen S, Noorthoorn EO, Collard RM, Comijs HC, Oude Voshaar RC, Naarding P. Value of physical activity and sedentary behavior in predicting depression in older adults. J Am Geriatr Soc 2016;64(March (3)):647-9.

[9] Wielopolski J, Reich K, Clepce M, Fischer M, Sperling W, Kornhuber J, et al. Physical activity and energy expenditure during depressive episodes of major depression. J Affect Disord 2015;15(March (174)):310-6.

[10] Hiles SA, Lamers F, Milaneschi Y, Penninx BWJH. Sit, step, sweat: longitudinal associations between physical activity patterns, anxiety and depression. Psychol Med 2017;47(June (8)):1466-77.
[11] Wassink-Vossen S, Collard RM, Oude Voshaar RC, Comijs HC, de Vocht HM, Naarding P. Physical (in)activity and depression in older people. J Affect Disord 2014;161(June):65-72.

[12] Rethorst $C D$, Trivedi $M H$. Evidence-based recommendations for the prescription of exercise for major depressive disorder. J Psychiatr Pract 2013;19(May (3)):204-12.

[13] Krogh J, Nordentoft M, Sterne JA, Lawlor DA. The effect of exercise in clinically depressed adults: systematic review and meta-analysis of randomized controlled trials. J Clin Psychiatry 2011;72(April (4)):529-38.

[14] Nguyen HQ, Koepsell T, Unutzer J, Larson E, LoGerfo JP. Depression and use of a health plan-sponsored physical activity program by older adults. Am J Prev Med 2008;35(August (2)):111-7.

[15] Silveira H, Moraes H, Oliveira N, Coutinho ES, Laks J, Deslandes A. Physical exercise and clinically depressed patients: a systematic review and metaanalysis. Neuropsychobiology 2013;67(2):61-8.

[16] Chi WC, Chang KH, Escorpizo R, Yen CF, Liao HF, Chang FH, et al. Measuring disability and its predicting factors in a large database in Taiwan using the World Health Organization Disability Assessment Schedule 2.0. Int J Environ Res Public Health 2014;11(November (12)):12148-61.

[17] Sargent-Cox KA, Butterworth P, Anstey KJ. Role of physical activity in the relationship between mastery and functional health. Gerontologist 2015;55 (February (1)):120-31.

[18] Penninx BW, Nolen WA, Lamers F, Zitman FG, Smit JH, Spinhoven P, et al. Twoyear course of depressive and anxiety disorders: results from the Netherlands Study of Depression and Anxiety (NESDA). J Affect Disord 2011;133(September $(1-2)): 76-85$.

[19] Comijs HC, Nieuwesteeg J, Kok R, van Marwijk HW, van der Mast RC, Naarding $\mathrm{P}$, et al. The two-year course of late-life depression; results from the Netherlands study of depression in older persons. BMC Psychiatry 201512 (February (15)) 20-015-0401-5.

[20] Penninx BW, Beekman AT, Smit JH, Zitman FG, Nolen WA, Spinhoven P, et al. The Netherlands Study of Depression and Anxiety (NESDA): rationale, objectives and methods. Int J Methods Psychiatr Res 2008;17(3):121-40.

[21] Comijs HC, van Marwijk HW, van der Mast RC, Naarding P, Oude Voshaar RC, Beekman AT, et al. The Netherlands study of depression in older persons (NESDO); a prospective cohort study. BMC Res Notes 20115(December (4)) 524-0500-4-524.

[22] Folstein MF, Folstein SE, McHugh PR. Mini-mental state. A practical method for grading the cognitive state of patients for the clinician. J Psychiatr Res 1975;12 (November (3)):189-98.

[23] Andrews G, Peters L. The psychometric properties of the composite international diagnostic interview. Soc Psychiatry Psychiatr Epidemiol 1998;33(February (2)):80-8.

[24] Wittchen HU, Burke JD, Semler G, Pfister H, Von Cranach M, Zaudig M. Recall and dating of psychiatric symptoms: test-retest reliability of time-related symptom questions in a standardized psychiatric interview. Arch Gen Psychiatry 1989;46(May (5)):437-43.

[25] Wittchen HU. Reliability and validity studies of the WHO-Composite International Diagnostic Interview (CIDI): a critical review. J Psychiatr Res 1994;28(January-February (1)):57-84.

[26] Rush AJ, Giles DE, Schlesser MA, Fulton CL, Weissenburger J, Burns C. The Inventory for Depressive Symptomatology (IDS): preliminary findings. Psychiatry Res 1986:18(May (1)):65-87.

[27] Rush AJ, Gullion CM, Basco MR, Jarrett RB, Trivedi MH. The Inventory of Depressive Symptomatology (IDS): psychometric properties. Psychol Med 1996;26(May (3)):477-86.

[28] Craig CL, Marshall AL, Sjostrom M, Bauman AE, Booth ML, Ainsworth BE, et al International physical activity questionnaire: 12-country reliability and validity. Med Sci Sports Exerc 2003;35(August (8)):1381-95.

[29] Tomioka K, Iwamoto J. Saeki K, Okamoto N. Reliability and validity of the International Physical Activity Questionnaire (IPAQ) in elderly adults: the Fujiwara-kyo Study. J Epidemiol Japn 2011;459-65.

[30] Patterson E. Guidelines for Data Processing and Analysis of the International Physical Activity Questionnaire (IPAQ). 2016 Available at: https://sites.google. $\mathrm{com} /$ site/theipaq/scoring-protocol [Accessed 19 July, 2017].

[31] Ainsworth BE, Haskell WL, Herrmann SD, Meckes N, Bassett Jr. DR, TudorLocke C, et al. Compendium of Physical Activities: a second update of codes and MET values. Med Sci Sports Exerc 2011;43(August (8)):1575-81.

[32] Kriegsman DM, Penninx BW, van EijkJT, Boeke AJ, Deeg DJ.Self-reports and general practitioner information on the presence of chronic diseases in community dwelling elderly. A study on the accuracy of patients' self-reports and on determinants of inaccuracy. J Clin Epidemiol 1996;49(December (12)):1407-17.

[33] Babor TF, Kranzler HR, Lauerman RJ. Early detection of harmful alcohol consumption: comparison of clinical, laboratory, and self-report screening procedures. Addict Behav 1989;14(2):139-57.

[34] Chwastiak LA, Von Korff M. Disability in depression and back pain: evaluation of the World Health Organization Disability Assessment Schedule (WHO DAS II) in a primary care setting. J Clin Epidemiol 2003;56(June (6)):507-14.

[35] Ustun TB, Chatterji S, Kostanjsek N, Rehm J, Kennedy C, Epping-Jordan J, et al. Developing the world health organization disability assessment schedule 2.0 Bull World Health Organ 2010;88(November (11)):815-23.

[36] Pearlin LI, Schooler C. The structure of coping. J Health Soc Behav 1978;19 (March (1)):2-21.

[37] de Beurs E, Comijs H, Twisk JW, Sonnenberg C, Beekman AT, Deeg D. Stability and change of emotional functioning in late life: modelling of vulnerability profiles. J Affect Disord 2005;84(January (1)):53-62. 
[38] Pearlin LI, Nguyen KB, Schieman S, Milkie MA. The life-course origins of mastery among older people. J Health Soc Behav 2007;48(June (2)):164-79.

[39] Thombs BD, Ziegelstein RC, Pilote L, Dozois DJ, Beck AT, Dobson KS, et al. Somatic symptom overlap in Beck Depression Inventory-II scores following myocardial infarction. Br J Psychiatry 2010;197(July (1)):61-6.

[40] Hagstromer M, Kwak L, Oja P, Sjostrom M. A 6 year longitudinal study of accelerometer-measured physical activity and sedentary time in Swedish adults. J Sci Med Sport 2015;18(September (5)):553-7.

[41] Ku PW, Fox KR, Chen LJ, Chou P. Physical activity and depressive symptoms in older adults: 11-year follow-up. Am J Prev Med 2012;42(April (4)):355-62.

[42] Franek J. Self-management support interventions for persons with chronic disease: an evidence-based analysis. Ont Health Technol Assess Ser 2013;13 (September (9)):1-60.
[43] Peckham AD, McHugh RK, Otto MW. A meta-analysis of the magnitude of biased attention in depression. Depress Anxiety 2010;27(December (12)):1135-42.

[44] Lee PH, Macfarlane DJ, Lam TH, Stewart SM. Validity of the International Physical Activity Questionnaire Short Form (IPAQ-SF): a systematic review. Int J Behav Nutr Phys Act 201121(October (8)) 115-5868-8-115.

[45] Soundy A, Roskell C, Stubbs B, Vancampfort D. Selection, use and psychometric properties of physical activity measures to assess individuals with severe mental illness: a narrative synthesis. Arch Psychiatr Nurs 2014;28(April (2)):135-51.

[46] Mota-Pereira J, Silverio J, Carvalho S, Ribeiro JC, Fonte D, Ramos J. Moderate exercise improves depression parameters in treatment-resistant patients with major depressive disorder. J Psychiatr Res 2011;45(August (8)):1005-11. 